\title{
Cell-Based Therapy for Neural Disorders - Anticipating Challenges
}

\author{
Arlene Y. Chiu • Mahendra S. Rao
}

Published online: 9 September 2011

(C) The American Society for Experimental NeuroTherapeutics, Inc. 2011

\begin{abstract}
Neurological syndromes, such as Alzheimer's disease, Parkinson's disease, multiple sclerosis, Huntington's disease, amyotrophic lateral sclerosis, and lysosomal storage disorders, such as Battens disease, are devastating because they result in increasing loss of cognitive and physical function. Sadly, no drugs are currently available to halt their progression. The relative paucity of curative approaches for these and other conditions of the nervous system have led to a widespread evaluation of alternative treatment modalities including cell-based interventions. Several cell types have been tested successfully in animal models where safety and efficacy have been demonstrated. Early clinical trials have also been initiated in humans, and some have shown a degree of success albeit on a more limited scale than in animal experiments. Recent demonstrations that pluripotent stem cells, such as embryonic stem cells and induced pluripotent stem cells, can differentiate into a variety of specific neural phenotypes has stimulated worldwide enthusiasm for developing cell-based intervention of neurological disease. Indeed, several groups are preparing investigational new drug applications to treat disorders as diverse as macular degeneration, lysosomal storage diseases, and Parkinson's disease. It is noteworthy that cell replacement therapies for neurological conditions
\end{abstract}

Electronic supplementary material The online version of this article (doi:10.1007/s13311-011-0066-9) contains supplementary material, which is available to authorized users.

A. Y. Chiu $(\bowtie)$

Beckman Research Institute of the City of Hope,

Duarte, CA, USA

e-mail: achiu@coh.org

M. S. Rao

Life Technologies Corporation,

Carlsbad, CA, USA face key challenges, some of which are unique, because of the development and organization of the nervous system, its metabolism, and connectivity. Choice of the cell (or cells), the process of manufacturing them, defining the delivery pathway, developing and testing in an appropriate preclinical model, selecting a patient population, and visualizing and following or monitoring patients all pose specific issues as related to the central and peripheral nervous systems. In this review, we address a myriad of challenges that are solvable, but require careful planning and attention to the special demands of the human nervous system.

Keywords Pluripotent stem cells $\cdot$ ESC $\cdot$ CNS roadblocks

\section{Introduction}

The characteristics and complexity of the human nervous system add several major challenges in considering cellbased therapies for neurological disorders. First there is the tremendous diversity of cellular phenotypes that make up the peripheral and central nervous systems. Second, this wide range of neurons are organized into complex networks that make up the functional infrastructure of a nervous system where the weakest link could abrogate or compromise the utility of the whole network, which may be responsible for a type of sensory input, a cognitive response, and/or a behavioral reaction. In humans, these intricate neuronal pathways are assembled and refined during a long period of development that extends into adolescence, and once established, these are used throughout life with little replacement. This limited ability to regenerate and self-renew as compared to other organs, such as skin, blood, and bone makes co-opting the normal repair process difficult. Finally, but not least important, 
replacing or grafting cells in the brain may be particularly difficult because access to the affected or target populations may require traversing and damaging vital systems, including the blood brain barrier, adjacent neuronal populations, and intervening circuits and pathways. Integration of new neurons into existing circuits may be very exacting, and ectopic clusters of grafted cells may impinge on and disturb other neural networks producing adverse behavioral consequences.

\section{The Right Cell for Each Therapy}

The human central nervous system (CNS) is comprised of as many as $10^{12}$ cells, the majority of which are fully differentiated and postmitotic. Approximately $10 \%$ of these cells are neurons, while the remaining ones are "support" cells, neighboring populations of glia that promote aspects of neuronal development, survival, and/or function.

Glial cells are made up of astrocytes, oligodendrocytes, and microglia. One class of glial cell, the astrocyte, provides trophic support and energy substrates necessary for neuronal function, while another, the oligodendrocyte, produces the myelin sheath that protects and insulates axons to enhance rapid communication between neurons. The neurons are organized with precision into circuits or networks that work in concert to regulate specific physical and mental activities. Any single neuron may make tens of thousands of connections with other neurons; some of these could be close to the cell body, while others could be at distances of several feet away. Neurons are functionally diverse, as well, in the types of neurotransmitter they make, the type of synapse they generate, and the number of connections and circuits they modulate. Thus, there is tremendous functional, morphological, and connectional diversity. Such diversity may extend to different classes of astrocytes and oligodendrocytes.

Part of the challenge in cell replacement therapy is to obtain replacement cells (i.e., neurons, glia, or an optimal, mixed population) with the precise phenotype (or phenotypes) or characteristics needed to restore the brain function that has been compromised by injury or disease, and then to deliver, insert, and integrate these cells into an appropriate, functional circuit. Thus, it may not be sufficient merely to produce large quantities of neurons that synthesize the correct neurotransmitter (e.g., acetylcholine to replace motor neurons lost to amyotrophic lateral sclerosis or cells that produce and release dopamine for Parkinson's disease). A cell population that is suitable for replacement may need to match much more closely their endogenous functional counterpart in all the parameters previously mentioned, and to be able to re-establish connectivity with existing networks in the patient's brain.
The mature mammalian brain does not routinely repair itself by regenerating new neurons, partly because stem or progenitor cell populations persist only in a few, select regions with few cues to direct their differentiation, migration, and integration into the existing appropriate and functional networks. Populations of adult neural stem cells (NSCs) are present primarily in the subventricular area around the lateral ventricle. Under normal conditions, they generate glia and a stream of neurons that are directed specifically to 2 regions of the brain, the olfactory bulb and the hippocampus. Although some controversy exists as to the number of stem cells present and to the extent of new neurogenesis, we believe that the total number of stem cells present in situ in the adult brain is small. These cells do not appear to participate in the repair process following injury or disease in most areas of the CNS, and their contribution appears to be limited to very specific regions in the adult nervous system. It is interesting to note that experience may regulate the activity and fate of these endogenous stem cells [1], although it is unclear how this property may be exploited to promote repair.

The lack of an ongoing repair process, coupled with the wide repertoire of neuronal phenotypes and their precise organization in circuits makes regeneration difficult in the mature nervous system. This is further complicated by the fact that the precise spatial connectivity of neural networks is established in stages during the course of a long period of time during development. As a result, areas of the brain become structurally different, each geared to perform distinct and unique functions using a precise subset of neuronal phenotypes with specific properties. These structural and functional differences emerge very early in development and appear to involve the local stem cells present in those regions. It is important to understand how NSCs acquired from different sources (such as various stages of development and/or regions of the CNS) will behave when reintroduced or transplanted into specific regions in the brain of the host. The choice of cell (or cells) for transplantation can be dictated by the age of the recipient, the disease process, and the response to injury. Neuronal circuits can be surprisingly flexible in adapting to injury and dysfunction [2]. Plasticity can occur on many levels, ranging from microscopic adjustments at the cellular and synaptic levels to massive re-routing and re-mapping of pathways in the brain. Plasticity also changes with age. For example, the high capacity for plasticity and repair in the brain of a child or young person becomes increasingly more restricted in adulthood. Recent studies show that endogenous agents can prevent neuronal plasticity in the mature CNS. The elimination of one such gene (lynx1) appears to extend the critical period during which the developing visual system retains the flexibility to adapt to experiential cues [3]. Restoration or enhancement of these forms of 
plasticity could promote the engagement of grafted neurons with endogenous networks that serve as substrates for the restoration of neurological performance.

Neuronal migration and the formation of circuits may be highly dependent on cues that are transiently expressed during certain stages of development, but are absent in the adult. It is becoming clear that the microenvironment or niche of the transplanted cells can regulate their fate and function. Myelin components have been shown to inhibit axonal sprouting following injury and may impede the ability of newly grafted neurons to form new circuits. In addition, the local inflammatory response in the transplant environment and the formation of astrocytic scars will influence the establishment of neuronal connectivity $[4,5]$. Therefore, understanding the roles of the microenvironment and developing strategies to regulate it may be critical to guide or drive the grafted cells to their desired phenotype to achieve a successful, behavioral outcome. Investigators are actively attempting to engineer this component of the graft to promote the survival, integration, and ultimately the function of the engrafted cells in the host.

\section{Possible Modes of Action of Engrafted Cells}

Stimulated, in part, by earlier efforts in tissue transplantation, researchers began in earnest in the 1990s to examine specific cell populations as potential sources for cell replacement for the nervous system. The range and repertoire of cells that have been evaluated has grown in the past 20 years to include fetal NSCs, adult NSCs, a variety of lineage specific precursor cells, neural cells transdifferentiated from other tissues $[6,7]$ or derived from pluripotent cells, such as embryonic stem cells (ESCs) and induced pluripotent stem cells (iPSCs). Several non-neural cell types and immortalized cell lines have also been used. Indeed, the range of cell populations tested in animal models is quite remarkable as is the evidence suggesting that successful functional recovery was achieved (for more detail see Lindvall and Bjorklund [8], and Lindvall and Kokaia [9]). Engraftment of exogenous cells is thought to help enhance repair in the nervous system by one or more of the mechanisms identified in Table 1. The proposed mechanism (or mechanisms) of action has a direct impact on a myriad of practical issues, including selection of the optimal cell (or cells) for transplantation, the dose and frequency of engraftment, the stage in the disease process at which to intervene, types of safety studies that should be performed, and the most suitable readouts of efficacy to apply.

Currently, a variety of cells, derived from a range of sources are being considered or tested in treatments for neurological disorders (summarized in Table 2). Aboody et
Table 1 Modes of action of cells transplanted to the nervous system

1. Replacement of missing, damaged, or dysfunctional cells

2. Enhancing neuronal survival

3. Supporting neurogenesis or neurite outgrowth

4. Promoting or restoring myelination

5. Release of trophic factors

6. Providing missing molecules

7. Mobilizing endogenous stem cells

8. Modulating scar formation and properties

9. Modulation of the immune response

10. Re-establishing the blood brain barrier

11. Vehicle for delivery of ex vivo gene therapy

12. Tracking and targeting cancerous cells for destruction

al. [10] provides an excellent review of ongoing clinical trials using stem cell-mediated approaches in the CNS (see Table 3). Although it is impossible to discuss each disease model in detail, certain generalizations are possible. In general, there is no simple template or decision tree that works for all cell therapies; decisions on the best type of cell to be used, the numbers or dose to be applied, and the optimal method of delivery will all have to be individually tailored to the disease condition. For example, to treat Parkinson's disease, the goal of cell therapy would be to restore and/or replace the damaged or lost dopaminergic neurons in the midbrain of patients. One approach might be to introduce primarily dopaminergic neurons to the exclusion of all other cells. It is likely that between 100,000 to 500,000 cells would be required, delivered into the parenchyma by injection, and be guided by magnetic resonance imaging. Although imaging following surgery indicated that grafts of fetal tissue succeeded in enhancing dopaminergic properties in the host brain, it is still unclear

Table 2 Cell types and sources used for treatments in the CNS

1. Neural stem cells derived from pluripotent stem cells, fetal tissue, and cadaveric tissue

2. Restricted progenitor cells (neuronal or glial restricted progenitors, oligodendrocytic progenitors)

3. Differentiated cells (motor and other neurons, retinal pigmented epithelium, astrocytes, oligodendrocytes, Schwann cells)

4. Re-purposed cells (Schwann cells, olfactory ensheathing glia, adrenal chromaffin cells, sympathetic ganglion cells)

5. Non-neural cells (mesenchymal stem cells, bone marrow stem cells, cord blood cells)

6. Combinatory therapies (mixed populations of cells, cells plus scaffold or extracellular matrix, genetically engineered cells for gene therapy or for growth and other factors)

$C N S=$ central nervous system 
Table 3 Companies and investigators engaged in recent cell therapies for the nervous system

1. ReNeuron - immortalized neural stem cell line for the treatment of stroke

2. Stem Cell Inc. - fetal neural stem cells for spinal cord injury and Batten's disease

3. Q Therapeutics - fetal glial precursors for myelin disorders

4. Geron Inc. - hESC-derived oligodendrocyte precursors for spinal cord injury

5. Advanced Cell Technology - hESC-derived retinal pigment epithelial cells for macular degenerative diseases

6. BrainStorm Cell therapeutics Inc. - autologous mesenchymal stem cells for ALS

7. Neuralstem Biopharmaceuticals - fetal neural stem cells for ALS

8. Karen Aboody (City of Hope) - immortalized neural stem cell line for brain tumors

9. Xianmin Zeng (Buck Institute) - hESC-derived dopaminergic neurons for Parkinson's disease

10. Larry Goldstein (UC, San Diego) - astrocyte precursors for ALS

11. Peter Coffey (London Project to Cure Blindness) - hESC-derived retinal pigment epithelium for macular degeneration

$A L S=$ amyotrophic lateral sclerosis; $h E S C=$ human embryonic stem cell

whether and how the transplanted cells function in this environment. The role of new dopaminergic neurons and how they will establish connectivity with the host's neural circuitry are key questions that have yet to be adequately addressed.

The numbers and type of cell (or cells) used would be very different if the strategy is to use the engrafted cells, stem cells, or their derivatives, as a delivery system dispensing one or more key growth factors to enhance the survival and function of existing neurons. In this case, migration and integration would not be required; inappropriate migration, such as homing to a stem cell niche, would be a disadvantage.

On the other hand, treating an injury to the CNS, such as spinal cord injury, traumatic brain injury, and stroke, would entail very different considerations regarding the numbers and phenotype of the cells used, as well as the mode and location of their delivery. If the intended therapy focuses on remyelination and/or halting the spread of secondary damage and scar formation, neuronal replacement may not be as important as glial replacement. The features of the desired cell (or cells) may include limited ability to mature toward a neuronal lineage and limited potential for migration other than toward a site of injury or lesion. For spinal cord injury, injection of cells via parenchymal or lumbar puncture may be an option, depending on where the damage occurred along the spinal cord. In stroke, if the lesion is large, perhaps intravenous or intra-arterial delivery may be contemplated, despite the known embolic risks. In this case, the ability of a cell to migrate through the blood vessel into the parenchyma and the ability to home to a target site may be highly desired properties.

NSCs appear to migrate far more freely in developing versus mature brains. This property has been exploited in designing treatments for lysosomal storage diseases, such as Batten's disease, in which the lack of a key protein has dire consequences in children. Once transplanted, the wild-type NSCs are expected to serve as pumps, delivering the missing enzymes widely throughout the developing brain.

Cell therapies that work by restoring a missing cell, enzyme, or protein require long-term survival of the engrafted cells; other approaches may not. NSCs can be engineered to deliver a lethal payload to brain tumors, functioning as suicide missions of seek and destroy. This strategy exploits the ability of NSCs to home selectively to select targets, such as cancerous cells, and once there, kill all cells in their vicinity. Therapeutic cells are delivered during neurosurgery following removal of the tumor to track and eliminate the last remaining tumorigenic cells, thus preventing the recurrence of the tumor. Given this mode of action, the transplanted cells are not required to survive for long in the host.

\section{Issues of High Importance to the Treatment of Neural Disorders}

Human cells have been in use in numerous therapies, such as bone marrow and cord blood transplantation for the treatment of cancer, implantation of pancreatic islets for type 1 diabetes, and mesenchymal cells for orthopedic indications. Many common issues dealing with cell transplantation have been addressed (for more detail see Carpenter et al. [11] and Ahrlund-Richter et al. [12]). We will focus on issues that are relevant to transplanting cells into the CNS. Table 3 summarizes recent and ongoing efforts by companies and investigators in academia to develop cell-based therapies for the nervous system.

\section{Delivery and Migration Issues}

In designing routes of administration, it is important to note that the brain is relatively insulated from the rest of the body. The CNS is protected structurally by a bony encasement, and chemically and immunologically protected by the glial limitans and the blood brain barrier, making it difficult to reach most areas of the brain and spinal cord without penetrating and causing damage to these structures. Some sites deep within the brain are hard to access by direct injection. Even minimally invasive techniques into the brain will result in some damage to neighboring neuronal populations or pathways. Alternate routes include 
introducing cells into the ventricles or cerebrospinal fluid space, or delivery via the circulatory system (arterial or venous injection) if the grafted cells are able to cross the blood brain barrier and home to the required sites within the CNS.

An attractive approach would be to stimulate the normal neurogenic regions within the host to produce the desired progenitor and differentiated cells for self-repair. Under this scheme, if migration is required, it must be kept in mind that in the adult, distances from endogenous stem cell niches to sites of injury are much larger than that encountered during development, and no direct pathways for cell migration exist in areas where there is no ongoing trafficking of cells. For example, radial glia that direct neuronal migration in the early developmental stages of the CNS are no longer present in the adult, and fiber tracts are oriented in directions that are not useful to migrating progenitor cells [13]. In attempting to home to a target, replacement cells may encounter barriers if pathways from the site of cell production or insertion to the site of injury are not well established. In addition, cells may not be able to distinguish between different types of lesions and may end up at ectopic sites. Thus, cell therapy in the brain faces what we euphemistically call a delivery problem.

\section{Mass Effect and Cell Death}

Once delivered, grafted cells encounter the dual problems of inability to survive or conversely undesired exuberance and excessive growth in their new environment in situ. Particularly in situations in which long-term survival of the graft is required in the host, apoptosis in large numbers abrogates the therapeutic function and benefits of the transplant. A great deal of attention is now being paid to promoting the longevity of transplanted cells, which includes providing a suitable microenvironment and the role of support cells for this purpose and attempting to enhance vasculogenesis of the graft. The other side of the coin of survival is the potential danger of uncontrolled proliferation resulting in ectopic clusters of cells and tumor formation. This could pose a significant problem in the CNS where established populations of neurons and axonal tracts are well organized and tightly apposed. The presence of and pressure created by a tumor, even if benign, could affect the normal function of these circuits.

\section{Cell Manufacture Issues}

An important point that needs thoughtful consideration is the number of cells required for the therapy in question, and the challenges in obtaining adequate quantities of purified, stable, and safe populations of such cells. The numbers of cells needed for a successful outcome can vary greatly. Although transplantation in some CNS diseases is envi- sioned to require approximately 10 million cells per patient, treatment of diabetes patients may require starting with a billion or more cells. Most primary cells (including adult stem cells) tend to grow in adherent culture and have relatively slow cycling times and a finite proliferative capacity. Further, maintaining karyotypic and epigenetic stability during long-term propagation is a challenge, and as such, cell therapy with stem cells still faces daunting problems in designing adequate manufacturing processes.

Several laboratories are turning to human ESCs (hESCs) and other pluripotent stem cells as a viable source of cells for transplantation, because in theory they have infinite proliferative capacity, and under appropriate conditions they can be coaxed to differentiate into any desired progenitor or mature phenotype. Nevertheless, investigators have found that pluripotent stem cells take time to properly mature, and so far this process of in vitro development cannot be accelerated to any great extent. Some approaches envision transplanting lineage progenitors to migrate to and mature within the host. It should be noted that the complex process of differentiation during development in vivo relies on positional information that may dictate the phenotype of cells and frequency of differentiation [14]. These types of environmental cues may not be present in the site of implant for cell therapies. Furthermore, as most diseases are not diseases of stem cells, but rather of differentiated populations, it is often transplants of specific cell types that are required. As a result, there needs to be developed protocols to direct differentiation of cells and equally importantly to create methods to select and enrich a desired population as part of the manufacturing process.

An issue that is often overlooked is the observation that cells age; stem cell population age after proliferating for long periods of time in vitro, and the aging of cells may also follow from the age of the organism from which the cells are derived. Aging affects the stability of the cell, its proliferative potential, and its ability to differentiate. Aging also results in the accumulation of DNA damage, both genomic and mitochondrial $[15,16]$, which in turn affects the quality of the stem cells and their reparative abilities, and may determine whether a particular cell therapy can be commercialized.

\section{Lineal and Positional Identity}

Another significant issue that is generally overlooked is that of acquisition and retention of lineal and positional identity. Current data suggest that this type of information is acquired early and this identity is maintained in culture and is difficult to reprogram. Pluripotent cells from early developmental stages that have not acquired such positional information may need appropriate cues to develop the identity desired for the therapy planned. On the other hand, 
primordial germ cells and adult-derived pluripotent stem cells may have acquired inappropriate information that may affect their ability to be used for certain purposes. This issue is significant in solid tissue organs where positional information may regulate fate, rate of proliferation, and phenotype specification. Spinal cord NSCs, for example, proliferate less and are biased to making motor neurons while midbrain-derived stem cells make dopaminergic neurons and NSCs from forebrain proliferate much more than either of the other populations of NSCs, and are biased toward making excitatory and inhibitory neurons. These different sources of NSCs have acquired positional information during their development that directs them toward different phenotypes.

\section{Issues Related to Cells as Delivery Vehicles}

As previously discussed, an obvious use of stem cells for replacement therapy is by transplanting either the stem cells directly or their derivatives, at a more mature stage following differentiation in vitro. A conceptually different approach is to use stem cells or progenitor cells as a vehicle to deliver trophic factors or specific signaling agents that direct the host tissue to initiate endogenous repair processes, or to restore a missing protein, such as an enzyme. StemCells, Inc. adopted this approach for the treatment of Battens disease, a rare but fatal lysosomal storage disorder that causes neurodegeneration in children, and has initiated a phase I clinical trial using human NSCs to deliver the missing enzyme (for more details see http://www.stemcellsinc.com/).

Genetic engineering techniques can be applied to stem cells to extend the expression of a desired molecule, or even introduce novel tools, such as a visualizing agent or a mediator of cytotoxicity. By harnessing the migratory and homing abilities of stem cells or some of their derivatives to transit from the circulation into the parenchyma of the brain and distribute widely or accumulate at sites of injury, investigators have been able to use such engineered cells to deliver a contrast agent that allows site-specific imaging or to provide a priming agent that enhances targeted cytotoxicity $[15,16]$.

Cells can also be engineered to introduce a foreign gene at a defined site or to knock out a defective gene, such as one responsible for autosomal dominant diseases. Such efforts are being undertaken to correct mutations that trigger Huntington's disease and the SOD1 mutations that cause amyotrophic lateral sclerosis (ALS). Alternatively, knock-in efforts are being designed to replace missing or functionally abnormal genes in a site-specific way. Such knock-in and knock-out technology is well developed in studies using mice, and several investigators have shown that these approaches can be used in both ESCs and iPSCs. Initially investigators were concerned that the low efficiency of these strategies may limit their usefulness, but several recent advances now suggest that the efficiency can be dramatically increased using transcription activator-like effectors, meganucleases, and integrases. It is not unreasonable to suspect that such repair and replacement strategies will soon become more commonplace. Indeed, several laboratories have shown that this is possible in mice and are working to extend the results to human cells.

The revolutionary discovery that somatic cells can be reprogrammed or induced to return to a pluripotent state now makes it technically possible to generate personalized pluripotent stem cells from an individual of virtually any age. Despite their obvious limitations, iPSCs offer one significant advantage in comparison with ESCs; because they originated from a cell taken from the host, they should not face immune rejection when grafted back into the same individual. It is therefore conceivable to create an iPSC line from a patient who suffers from a gene defect, correct the defect by using genetic engineering methods, differentiate the "corrected" cell line into the desired cells of choice, and finally transplant these genetically correct, differentiated cells back into the patient for an effective cure. Although technically feasible, there remain several hurdles to overcome before this strategy becomes practical. Nevertheless, given the rapid pace of discovery in this field, perhaps with some optimism it can be suggested, that such efforts could succeed within this decade.

If the correction or repair is made in a stem cell population that replaces the endogenous, defective stem cells, the cure could essentially be a permanent one. Stem cells offer the ability to perform the repair ex vivo, amplify and obtain sufficient numbers of the repaired cell, which are then delivered to a niche suitable for permanent engraftment and survival. Since these are autologous stem cells, such an approach offers the advantage of bypassing issues of immune rejection.

Instead of repairing a defect or transferring functional genes into the cell, a related approach disrupts gene activity by homologous recombination. This might be an effective strategy in cases in which dominant mutant alleles are responsible for pathology (e.g., familial ALS or Huntington's disease), or in cases in which diminution of gene expression might be desirable for other reasons. One barrier to gene knockout therapy is the low frequency of homologous recombination in most somatic cells. However, ESCs have relatively high rates of homologous recombination, a property that has served mouse geneticists well for reconstitution of mutant mice with disrupted genes. If human ESCs exhibit the same property, production of human knockout ESCs should be possible. Indeed recent publications have shown that targeting by homologus recombination is feasible and with similar frequency as seen in mouse ESCs in which this type of repair has been well documented. Indeed the concept of starting with an 
adult stem cell from an animal carrying a gene defect, reprogramming it to induce pluripotency, and then repairing the defect using homologous recombination or gene targeting has been shown to be feasible [17]. It is not unreasonable to assume that such a repair should be feasible in humans as well. For more details see Xue et al. [18]. As with other gene manipulation approaches, several improvements have been reported with homologous recombination, and strategies to perform repairs have evolved. These include using zinc finger nucleases, other nucleases, integrases, and other methods for DNA repair. A detailed discussion of these types of alternate technologies is outside the scope of this chapter and can be found in specialized reviews on this subject (for more detail see Zeng and Rao [19] and Kamb [20], and the references therein).

\section{Immune Issues}

The CNS has been described as an immune privileged organ where the external glial limitans and blood brain barrier act as physical barriers guarding against entry of the molecules and cells, including inflammatory molecules and immune cells. This may enable engraftment of potentially immunogenic cells, if they can cross the blood brain barrier. Unfortunately, the process of inserting or grafting cells directly into the CNS often involves breaching these protective layers, resulting in a local inflammatory response that can destroy and deplete the reservoir of newly grafted cells. Furthermore, even if cells survive the initial damage and the blood brain barrier is restored, other inflammatory episodes or secondary damage responses can cause a delayed loss of engrafted cells with a resultant loss of benefit.

Immune matching is difficult, and even when human leukocyte antigen (HLA) types are matched, some immune suppressive therapy is required. It should be noted, however, that in the clinical studies where fetal tissue was transplanted for the treatment of Parkinson's disease, immunosuppressive therapy was discontinued after a year or so with little evidence of loss of graft in patients. It remains to be determined what the optimum regime will be for immunosuppression and how long it will have to be maintained.

A second equally important issue with the immune response is in performing animal experiments. Here the xenograft rejection response bears no relationship with the disease and repair process that the investigators are trying to model, and suppressing the immune response in this situation may not allow them to determine the true viability of the graft in humans. Many attempts have been made to address these issues from generating models with a humanized immune system to performing parallel rat into rat or primate into primate studies. None of these have been optimal because it is unclear whether equivalent stages of cells in different species behave identically.
More recently, the ability to generate personalized iPSCs presents the potential of bypassing the immune issue altogether; because iPSCs are derived from the host, they could be a rich source of autologous, "custom-made" cellular products for the recipient. One concern revolves around the significant costs of such an approach and introduces additional regulatory hurdles as each new cell product line may have to be qualified as being safe or comparable to previously used cells. An unforeseen issue came from a recent study in which mouse iPSCs unexpectedly triggered a strong T-cell response in their matched murine hosts [21]. Further investigations will be required to clarify the source of this immunogenicity.

\section{Preclinical Animal Models}

Most investigators preparing to test a product or process in patients need to file an application for an Investigational New Drug (IND) with the Food and Drug Administration. The Food and Drug Administration requires strong evidence of preclinical studies that demonstrate safety and efficacy of a treatment before permitting a novel cell-based therapy to be tested in patients. Animal models play a critical role in testing for feasibility and bioactivity, and for predicting efficacy and dosage. The design and selection of animal models is therefore intimately related to the therapeutic question that is being asked.

Detection of tumorigenic cells is a key safety issue with stem cell therapies involving products derived from pluripotent stem cells, because such cells, if undifferentiated produce teratomas following transplantation. Because mice have a short lifespan of approximately 9 months, this length of time might not suffice to determine whether a cell population will eventually produce tumors following transplantation in humans. One solution is to carry out serial transplantations where grafted cells are transplanted into multiple animals with time. For example, the cells to be tested are introduced into the kidney capsule of a first mouse; later the whole kidney is transplanted into a second, younger mouse, and then a third to increase the total test period. Serial transplantation has a very high success rate. Safety in the method of cell delivery is also assessed first in preclinical, animal studies in which cells should be introduced into the same site, using the same route and technique as proposed for patients.

A major roadblock to developing cell therapies is the availability and use of appropriate animal models, especially large or humanized animal models for testing safety and efficacy, and the related issue of xenografts. The key is that the animal model(s) need to be chosen to generate the best preclinical data that supports the approach proposed. Small animal models have the benefit of being easy to use, but may not be very robust for predicting outcomes in clinical 
trials. A common strategy is to conduct functional tests in small mammals and to turn to larger animals, such as the pig, to test the use of devices or delivery systems. Large animals may also be best for testing dosing regimes while smaller mammals are best for assessing efficacy. The most nagging problem in using animal models for testing human cells is the "xeno" factor: that is, when human cells are tested in any animal model, the graft is always a xenograft and subject to additional immunological challenges. Yet there are few alternatives for testing the human candidate cells for efficacy. The real value of humanized animal models is unclear, because they have not been used extensively.

Rational approaches for selecting animal models for neurological conditions require an understanding of the nature of the disorder. A relevant animal model should show behavioral deficits that resemble symptoms in patients. Some animal models reproduce the human condition quite faithfully, as in the case of familial ALS in which the same mutations that are found in human disease produces a similar progressive loss of neurons and motor function in dogs [22], rats [23], and mice [24]. Models of injury, such as spinal cord injury and stroke, can be induced in animals and are used extensively to evaluate treatments for both acute and chronic dysfunction in patients. Unfortunately, the etiologies of many neurological diseases are not understood, making it difficult to find the most relevant models. Some animal models only replicate the cellular loss, but not the mechanism underlying the pathology. Inducing the acute destruction of dopaminergic neurons by chemical means does not replicate the gradual and progressive loss of these same neurons with time that is the hallmark of Parkinson's disease. There are limitations in the best of animal models available, which need to be considered in making strategic choices.

\section{Preparing for Clinical Trials}

Clinical assessment and endpoints can be very different for acute versus chronic disease, or for different stages of a progressive or invasive disease. The usefulness and success of a clinical trial in that it provides new and interpretable data depends greatly on the clinical endpoints used. In many neurological disorders, there is a need for greater effort in developing these tools or measures for evaluating recovery in patients.

Another important point involves the criteria used for patient selection. In situations in which some current treatments are available, the bar is set very high for testing new and innovative, but risky, approaches for this condition; therefore, these trials should only target patients with a poor prognosis for survival, such as the most advanced cases and those who are severely disabled.
There is a big difference between the United States and Europe regarding the conduct of randomized, double-blind, placebo-controlled trials. In general, in Europe these trials are considered unethical given the high risk of the procedures to patients, whereas clinicians in the United States find it difficult to interpret and accept the results from open-label trials conducted without controls. Finally, the long-term follow-up of patients poses yet another challenge because of lack of funding for this aspect of the trial.

\section{Summary and Future Directions}

Efforts to use cells in several therapeutic indications in the nervous system are underway. These include using differentiated cells, such as retinal pigment epithelium for macular degeneration [25], or progenitor cell lines by ReNeuron and by NeuralStem for stroke, by Stem Cell Inc. for Batten's Disease, or by the Aboody team to target cancer in the brain $[26,27]$. In general, these investigators have identified the problem, identified the appropriate cell to use and defined a delivery mechanism for the purpose. Some general principles can be elucidated from their efforts. First, therapy is generally local or focal for diseases that affect specific regions of the brain or specific populations of cells. Thus far, investigators have been unable to effectively co-opt the limited endogenous repair programs present in the adult CNS, and efforts to use non-neural cells, either as immune modulators or for their transdifferentiation abilities, have met with limited success. Second, as a better understanding of the mechanisms underlying each disease has emerged, investigators have found it necessary to develop newer and more relevant animal models. Many investigators are concluding that they also need better tools with which to evaluate the behavioral effects that characterize or accompany different disorders of the CNS. In addition, all investigators using human cells for therapy must deal with the "xeno" issue in their preclinical animal studies. Third, it became clear that optimal delivery of cells required the development of novel scaffolds or very specialized devices, such as those for the spinal cord injury.

Many challenges remain, and there is no doubt that future efforts will be focused on the issues we have highlighted. New advances in genetic engineering and methodologies for directing differentiation, the development of better animal models, improvements in scale-up technologies, cell purification and manufacturing, and reduction in the cost of developing iPSCs for personalized treatments will all, in our opinion, improve the quality and efficacy of these early efforts. By reducing the cost, these may make such experimental therapies practical as the future standard of care for the intractable conditions of today. 
Acknowledgment Full conflict of interest disclosure is available in the electronic supplementary material for this article.

\section{References}

1. Dranovsky A, Picchini AM, Moadel T, et al. Experience dictates stem cell fate in the adult hippocampus. Cell 2011;70:908-923.

2. Wieloch T, Nikolich K. Mechanisms of neural plasticity following brain injury. Curr Opinion in Neurobiology 2006;16:258-264.

3. Morishita H, Miwa JM, Heintz N, Hensch TK. Lynx1, a cholinergic brake, limits plasticity in adult visual cortex. Science 2010;330:1238-1240.

4. Busch SA, Hamilton JA, Horn KP, et al. Multipotent adult progenitor cells prevent macrophage-mediated axonal dieback and promote regrowth after spinal cord injury. J Neurosci 2011:31:944-953.

5. Horn KP, Busch SA, Hawthorne AL, van Rooijen N, Silver J. Another barrier to regeneration in the CNS: activated macrophages induce extensive retraction of dystrophic axons through direct physical interactions. J Neurosci 2008;28:9330-9341.

6. Pfisterer U, Kirkeby A, Torper O, et al. Direct conversion of human fibroblasts to dopaminergic neurons. PNAS USA 2011;108:10343-10348.

7. Vierbuchen T, Ostermeier A, Pang ZP, Kokubu Y, Sudhof TC, Wernig M. Direct conversion of fibroblasts to functional neurons by defined factors. Nature 2010;463:1035-1041.

8. Lindvall O, Bjorklund A. Cell therapy in Parkinson's disease. NeuroRx 2004;1:382-393.

9. Lindvall O, Kokaia Z. Prospects of stem cell therapy for replacing doamine neurons in Parkinson's disease. Trends Pharmacol Sci 2009;30:260-267.

10. Aboody K, Capela A, Niazi N, Stern JH, Temple S. Translating stem cell studies to the clinic for CNS repair: current state of the art and the need for a Rosetta Stone Neuron 2011;70:597-613.

11. Carpenter MK, Frey-Vasconcells J, Rao MS. Developing safe therapies from human pluripotent stem cells. Nature Biotech 2009;27:606-613.

12. Ahrlund-Richter L, De Luca M, Marshak DR, Munsie M, Veiga A, Rao M. Isolation and production of cells suitable for human therapy: challenges ahead. Cell Stem Cell 2009;4:20-26.
13. Rao M. Conserved and divergent paths that regulate self-renewal in mouse and human embryonic stem cells. Developmental Biol 2004;275:269-286.

14. Gilbert S. The morphogenesis of evolutionary developmental biology. Int J Dev Biol 2003;47:467-477.

15. Maitra A, Arking DE, Shivapurkar N, et al. Genomic alterations in cultured human embryonic stem cells. Nature Genet 2005;37:1099 1103.

16. Zeng X, Rao MS. Human embryonic stem cells: long term stability, absence of senescence and a potential cell source for neural replacement. Neuroscience 2007;145:1348-1358.

17. Zwaka TP, Thomson JA. Homologous recombination in human embryonic stem cells. Nature Biotech 2003;21:319-321.

18. Xue H, Wu S, Papadeas ST, et al. A targeted neuroglial reporter line generated by homologous recombination in human embryonic stem cells. Stem Cells 2009;27:1836-1846.

19. Zeng X, Rao MS. Controlled genetic modification of stem cells for developing drug discovery tools and novel therapeutic applications. Curr Opin Mol Ther 2008;10:207-213.

20. Kamb A. Consequences of nonadaptive alterations in cancer. Mol Biol Cell 2003:14:2001-2005.

21. Zhao T, Zhang ZN, Rong Z, Xu Y. Immunogenicity of induced pluripotent stem cells. Nature 2011;474:212-216.

22. Awano T, Johnson GS, Wade CM, et al. Genome-wide association analysis reveals a SOD1 mutation in canine degenerative myelopathy that resembles amyotrophic lateral sclerosis. PNAS USA 2009;106:2794-2799.

23. Matsumoto A, Okada Y, Nakamichi M, et al. Disease progression of human SOD1 (G93A) transgenic ALS model rats. J Neurosci Res 2006;83:119-133.

24. Gurney $\mathrm{ME}, \mathrm{Pu} \mathrm{H}$, Chiu AY, et al. Motor neuron degeneration in mice that express a human $\mathrm{Cu}, \mathrm{Zn}$ superoxide dismutase mutation. Science 1994;264:1772-1775.

25. Huang Y, Enzmann V, Ildstad ST. Stem cell-based therapeutic applications in retinal degenerative diseases. Stem Cell Rev 2011;7:434-445.

26. Frank RT, Najbauer J, Aboody KS. Concise review: stem cells as an emerging platform for antibody therapy of cancer. Stem Cells 2010;28:2084-2087.

27. Gutova M, Najbauer J, Chen MY, Potter PM, Kim SU, Aboody KS. Therapeutic targeting of melanoma cells using neural stem cells expressing carboxylesterase, a CPT-11 activating enzyme. Curr Stem Cell Res Ther 2010;5:273-276. 